\title{
Signal Processing Techniques for Reducing the Impact of Fiber Nonlinearities on System Performance
}

\author{
J. C. Cartledge ${ }^{(1)}$, A. D. Ellis ${ }^{(2)}$, A. Shiner ${ }^{(3)}$, A. I. Abd El-Rahman ${ }^{(1)}$, M. E. McCarthy ${ }^{(2)}$, \\ M. $\operatorname{Reimer}^{(3)}$, A. Borowiec ${ }^{(3)}$, A. Kashi ${ }^{(1)}$ \\ ${ }^{(1)}$ Queen's University, Kingston, ON, Canada; ${ }^{(2)}$ Aston University, Birmingham, United Kingdom; ${ }^{(3)}$ Ciena, Ottawa, ON, Canada \\ john.cartledge@queensu.ca
}

\begin{abstract}
Signal processing techniques for mitigating intra-channel and inter-channel fiber nonlinearities are reviewed. More detailed descriptions of three specific examples highlight the diversity of the electronic and optical approaches that have been investigated.
\end{abstract}

\section{Introduction}

Intra-channel and inter-channel fiber nonlinearities are major impairments in coherent transmission systems that limit the achievable transmission distance [1]. Consequently, a wide variety of signal processing techniques for mitigating the performance implications of fiber nonlinearities have been investigated. Key distinguishing features of these techniques are their complexities, their capabilities to mitigate intra-channel or inter-channel nonlinearities, and whether the implementation uses digital signal processing and/or all-optical signal processing. An important challenge is to achieve useful improvements in system performance with acceptable levels of implementation complexity.

In broad terms, the techniques for reducing the impact of fiber nonlinearities on system performance include those that use compensation of the nonlinearity-induced signal distortion and those that make signal propagation more tolerant to fiber nonlinearities. The former category includes (i) single-channel and multi-channel digital back propagation using the coupled nonlinear Schrödinger equation (CNLSE) [2,3], (ii) Volterra series nonlinear equalizers [4], (iii) perturbation solutions to the CNLSE [5-7], (iv) compensation of the nonlinear phase noise [8], and (v) optical phase conjugation [9]. The latter category includes (i) optical pulse shaping [10], (ii) subcarrier modulation [11], (iii) nonlinear noise squeezing that occurs for real-valued signals [12] or conjugated twin waves in the polarization domain [13], and (iv) constellation design [14-16]. Signal processing techniques for mitigating fiber nonlinearities are reviewed with specific examples illustrating the diversity of the techniques that have been explored.

\section{Pre-compensation for intra-channel fiber nonlinearities}

The perturbation-based pre-compensation technique compensates accumulated nonlinearities with only one computation step and can be implemented with one sample per symbol [5]. However, calculation of the nonlinear perturbation involves single and double summations that are functions of the transmitted symbol sequence and perturbative expansion coefficients $\left\{C_{m, n}\right\}$. The required number of terms in the summations can be reduced by using symmetric electronic dispersion compensation (SEDC) and root-raised-cosine (RRC) pulse shaping [7]. With SEDC two simplifications result: (i) the real parts of the $\left\{C_{m, n}\right\}$ coefficients are zero and (ii) the imaginary parts are calculated based on half the link length. In combination with a RRC pulse shape, this reduces the dispersion induced pulse spreading and hence the required number of terms in the truncated summations.

For a $128 \mathrm{Gbit} / \mathrm{s}$ DP 16-QAM signal and transmission over $3600 \mathrm{~km}$ of standard single mode fiber with EDFA amplification, the dependence of the BER on launch power is shown in Fig. 1 for linear post-compensation for dispersion (LC), symmetric linear pre- and post-compensation for dispersion (LC-SEDC), and RRC-SEDC nonlinear pre-compensation. The roll-off factor for the RRC pulse shape was 0.1 and the number of terms in the truncated summations for the RRC-SEDC algorithm was based on $20 \log _{10}\left|C_{m, n} / C_{0,0}\right|>-35 \mathrm{~dB}$. The dependence of the BER at optimum launch power on fiber length for the three algorithms is shown in Fig. 2. For a forward error correction (FEC) coding bit error ratio (BER) threshold of 0.02, transmission over $4200 \mathrm{~km}$ of fiber was achieved with RRCSEDC nonlinear pre-compensation, an increase of $900 \mathrm{~km}$ relative to LC and LC-SEDC, a net benefit of around $1.2 \mathrm{~dB}$.

\section{Constellation formats tolerant to inter-channel fiber nonlinearities}

The simultaneous in-phase and quadrature modulation of two orthogonally polarized carriers may be referred to as four-dimensional (4D) modulation [16]. By defining constellation symbols based on 4D modulation of two adjacent symbol periods, eight-dimensional (8D) modulation is obtained [14, 15]. An 8D power and polarization balanced modulation format (denoted the $\mathrm{X}$-constellation) is related to the biorthogonal format by a real-valued $8 \mathrm{D}$ rotation which preserves Euclidian distance and is designed to provide a constant power and zero degree of polarization in all $8 \mathrm{D}$ symbol periods [13]. This has been used to reduce cross-polarization modulation in dispersion managed systems as a result of zeroing the degree of polarization. 


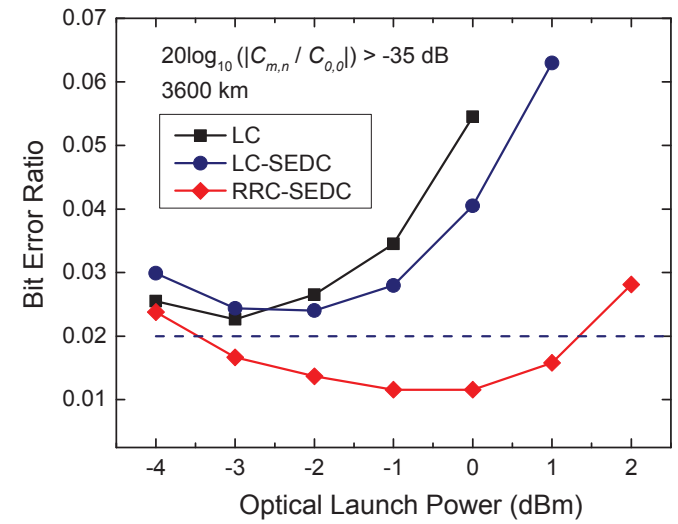

Fig. 1. Dependence of the BER on the optical launch power for a fiber length of $3600 \mathrm{~km}$.

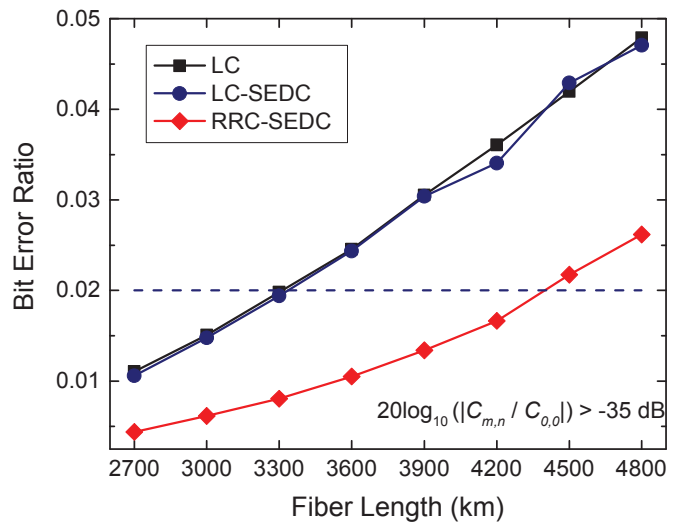

Fig. 2. Dependence of the BER at optimum launch power on the fiber length.

Fig. 3 illustrates the dependence of the BER on electrical signal-to-noise ratio (simulated back-to-back 35 Gbaud system) for the four-dimensional DP BPSK, 8D biorthogonal, and X-constellation modulation formats. All these formats operate at a spectral efficiency of two bits per dual polarization signaling interval (time slot). The dependence of the relative net system margin on launch power is shown in Fig. 4 for the center channel of a 9 channel 35 Gbaud system (40 GHz channel spacing) propagating over $50 \times 80 \mathrm{~km}$ spans of E-LEAF fiber with $90 \%$ inline compensation at each repeater. The closed and open symbols show the largest and smallest relative margins as the polarization states of the interfering channels at the input of the system are varied with respect to the center channel. The power and polarization balanced X-constellation format is virtually independent of the polarization states of the neighboring channels; the margin improves by $1.8 \mathrm{~dB}$ relative to DP BPSK with optimum polarization alignment.

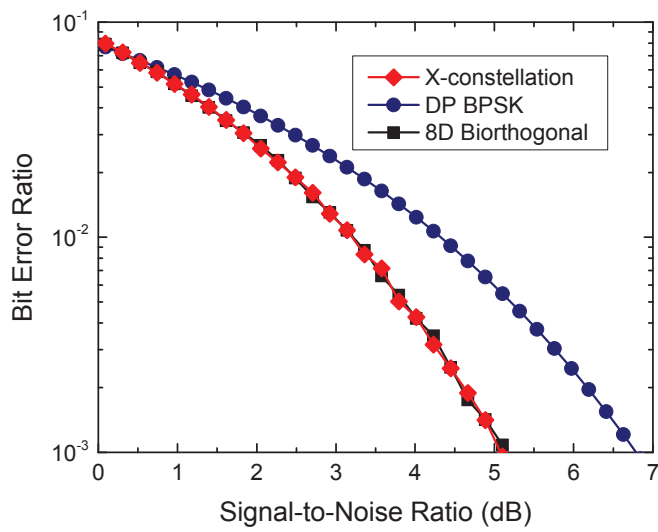

Fig. 3. Dependence of the bit error ratio on the electrical signal-tonoise ratio.

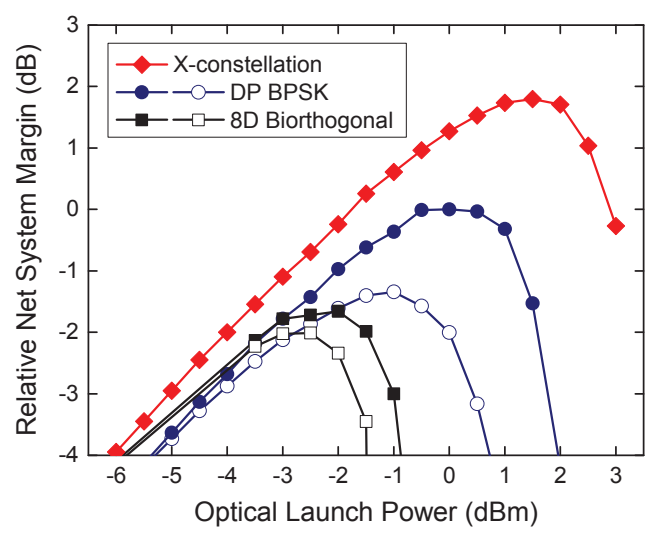

Fig. 4. The net system margin relative to the maximum system margin.

\section{Optical phase conjugation and digital backpropagation}

Mitigation of the effects of fiber nonlinearities using digital signal processing is eventually limited by stochastic effects such as amplified spontaneous emission noise and polarization mode dispersion (PMD) [17]. A simple modification is to add an optical phase conjugator (OPC) close to the mid-point of a link, which compensates for deterministic nonlinearities and disrupts the accumulation of parametric noise amplification [9]. It is conceivable that conditions such as dispersion-power symmetry could be satisfied using appropriate Raman amplified links [18], but locating an OPC at the exact mid-point would be difficult. However, since OPC compensates for the bulk of the nonlinear impairments, it may be readily combined with digital back propagation (DBP) to compensate for the residual nonlinearity [19]. The impact of the OPC being offset slightly from the center of the link on the performance of a 2100 $\mathrm{km}$ DP 64-QAM system (75 km spans) is shown in Fig. 5. The BER is plotted for systems with LC, DBP with an OPC offset from the mid-point of the link and different levels of DBP accuracy. Without DBP exact OPC mid-point placement is essential while $99.99 \%$ accurate DBP allows the OPC to be displaced more than $350 \mathrm{~km}$ from the midpoint. The combination of OPC/DBP relaxes both the required DBP accuracy and the OPC placement, and offers a performance benefit. The complexity/accuracy trade-off is summarized in Fig. 6 where the required DBP complexity to accommodate an OPC offset from link mid-point by one span is plotted as a function of fiber length for a variety 
of modulation formats (target BER of $10^{-3}$ ). Substantial performance benefit may be retained even if the OPC is displaced from the mid-point by a whole span provided the DBP accuracy exceeds around $90 \%$.

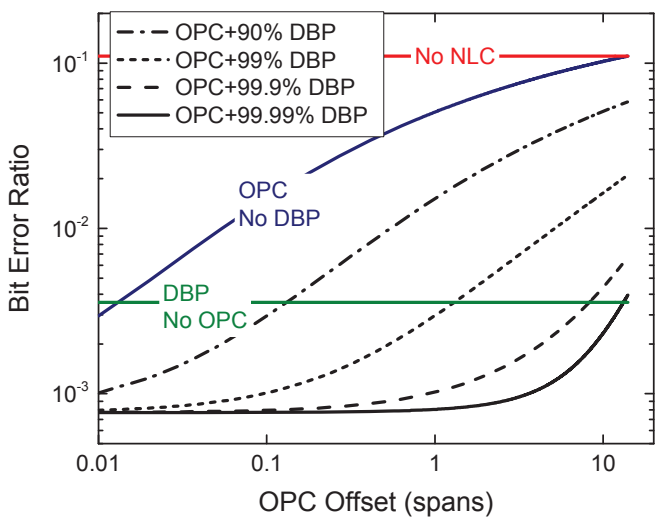

Fig. 5. Dependence of the bit error ratio on the OPC offset from link mid-point for a range of DBP performance levels.

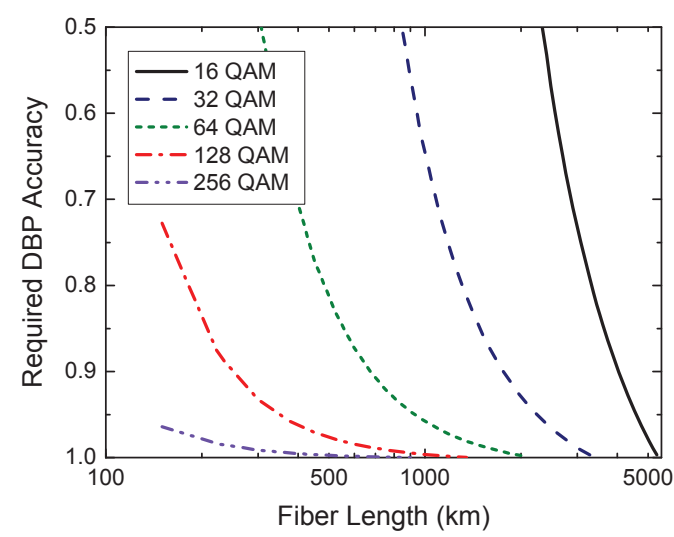

Fig. 6. Minimum required DBP accuracy to accommodate an OPC offset from link mid-point by one span.

\section{Summary}

Electronic and optical techniques have been considered to illustrate the diversity of approaches that have been investigated for mitigating the effects of fiber nonlinearities on system performance.

\section{Acknowledgement}

We thank Ying Gao, Maurice O'Sullivan, Charles Laperle and Kim Roberts for their contributions to this paper. A. Ellis and M. McCarthy thank EPSRC for funding part of this work through grant agreement EP/L000091/1 (PEACE).

\section{References}

[1] A. Mecozzi and R.-J. Essiambre, "Nonlinear Shannon limit in pseudolinear coherent systems," J. Lightw. Technol. 30, 2011-2024 (2012).

[2] A. Napoli, Z. Maalej, V. A. J. M. Sleiffer, M. Kuschnerov, D. Rafique, E. Timmers, B. Spinnler, T. Rahman, L. D. Coelho, and N. Hanik, "Reduced complexity digital back-propagation methods for optical communication systems," J. Lightw. Technol. 32, 1351-1362 (2014).

[3] R. Maher, D. Lavery, D. Millar, A. Alvarado, K. Parsons, R. Killey and P. Bayvel, "Reach enhancement of 100\% for a DP-64QAM superchannel using MC-DBP,” Proc. Conf. Opt. Fiber Commun., Th4D.5 (2015).

[4] F. P. Guiomar and A. N. Pinto, "Simplified Volterra series nonlinear equalizer for polarization-multiplexed coherent optical Systems," J. Lightw. Technol. 31, 3879-3891 (2013).

[5] Z. Tao, L. Dou, W. Yan, L. Li, T. Hoshida, and J. C. Rasmussen, "Multiplier-free intrachannel nonlinearity compensating algorithm operating at symbol rate," J. Lightw. Technol. 29, 2570-2576 (2011).

[6] X. Liang and S. Kumar, "Multi-stage perturbation theory for compensating intra-channel nonlinear impairments in fiber-optic links," Opt. Exp. 22, 29733-29745 (2014).

[7] Y. Gao, J. C. Cartledge, A. S. Karar, S. S.-H. Yam, M. O’Sullivan, C. Laperle, A. Borowiec, and K. Roberts, "Reducing the complexity of perturbation based nonlinearity pre-compensation using symmetric EDC and pulse shaping," Opt. Exp. 22, 1209-1219 (2014).

[8] Z. Tao, L. Li, L. Liu, W. Yan, H. Nakashima, T. Tanimura, S. Oda, T. Hoshida, and J. C. Rasmussen, "Improvements to digital carrier phase recovery algorithm for high-performance optical coherent receivers," IEEE J. Sel. Topics Quant. Electron. 16, 1201-1209, (2010).

[9] A. D. Ellis, M. E. McCarthy, M. A. Z. Al-Khateeb, and S. Sygletos, "Capacity limits of systems employing multiple optical phase conjugators", Opt. Exp. 23, 20381-20393 (2015).

[10] A. S. Karar, S. Gazor, Y. Gao, J. C. Cartledge, M. O’Sullivan, C. Laperle, A. Borowiec, and K. Roberts, "Mitigating intra-channel nonlinearity in coherent optical communications using ISI-free polynomial pulses," IEEE Photon. Technol. Lett. 27, 1653-1655 (2015).

[11] P. Poggiolini, A. Carena, Y. Jiang, G. Bosco, and F. Forghieri, "On the ultimate potential of symbol-rate optimization of increasing system maximum reach", Proc. Eur. Conf. Opt. Commun., We.4.6.2 (2015).

[12] X. Liu, S. Chandrasekhar, P.J. Winzer, R. W. Tkach, and A. R. Chraplyvy, “406.6-Gb/s PDM-BPSK superchannel transmission over 12,800km TWRS fiber via nonlinear noise squeezing,” Proc. Conf. Opt. Fiber Commun., PDP5B.10 (2013).

[13] X. Liu, A. R. Chraplyvy, P. J. Winzer, R. W. Tkach, and S. Chandrasekhar, "Phase-conjugated twin waves for communication beyond the Kerr nonlinearity limit," Nat. Photon. 7, 560-568 (2013).

[14] A. Shiner, M. Reimer, A. Borowiec, S. Oveis Gharan, J. Gaudette, P. Mehta, D. Charlton, K. Roberts, M. O'Sullivan, "Demonstration of an 8-dimensional format with reduced inter-channel nonlinearities in polarization multiplexed coherent systems," Opt. Exp. 22, 20366-20374 (2014).

[15] M. Reimer, S. Oveis Gharan, A. D. Shiner, A. Ghasemi, and M. O'Sullivan, "Performance optimized modulation formats in 4 and 8 dimensions," Proc. Sig. Proc. Photon. Commun., SpT3D.6 (2015).

[16] A. Alvarado and E. Agrell, "Four-dimensional coded modulation with bit-wise decoders for future optical communications," J. Lightw. Technol. 33, 1993- 2003 (2015).

[17] T. Xu, A. Alvarado, R. I. Killey, and P. Bayvel, "On the performance of multichannel digital backpropagation in high-capacity long-haul optical transmission," Opt. Exp. 22, 30053-30062 (2014).

[18] A. D. Ellis, I. D. Phillips, M. Tan, M. F. C. Stephens, M. E. McCarthy, M. A. Z. Al Kahteeb, M. A. Iqbal, A. Perentos, S. Fabbri, V. Gordienko, D. Lavery, et al., "Enhanced superchannel transmission using phase conjugation,” Proc. Eur. Conf. Opt. Commun., We.2.6.4 (2015). [19] A. D. Ellis, S. T. Le, M. A. Z. Al-Khateeb, S. K. Turitsyn, G. Liga, D. Lavery, T. Xu, and P. Bayvel, "The impact of phase conjugation on the nonlinear-Shannon limit," IEEE Summer Top. Mtg, TuF3.2, (2015). 